Vásquez-Bardales, J., Vargas-Zelada, Y.N., Pinedo-Jiménez, J., García-Ruiz, A., Valderrama-Freyre, H., \& RamírezHernandez, J.J. (2021). Alimentación, comportamiento de oviposición, ciclo de vida y enemigos naturales de Hamadryas feronia (Nymphalidae) en la Amazonía del Perú. Revista de Biología Tropical, 69(2), 524-533. DOI 10.15517/rbt.v69i2.44969

DOI 10.15517/rbt.v69i2.44969

\title{
Alimentación, comportamiento de oviposición, ciclo de vida y enemigos naturales de Hamadryas feronia (Nymphalidae) en la Amazonía del Perú
}

Joel Vásquez-Bardales ${ }^{1 *}$; (D) Orcid: 0000-0002-0584-7310

Yurisa Norelli Vargas-Zelada ${ }^{1}$; (D) Orcid: 0000-0002-7166-1223

Julio Pinedo-Jiménez²; (D) Orcid: 0000-0001-6784-0803

Alberto García-Ruiz ; (D) Orcid: 0000-0002-3827-0036

Heiter Valderrama-Freyre ${ }^{4}$; (D) Orcid: 0000-0002-6177-2755

Juan José Ramírez-Hernandez ; (D) Orcid: 0000-0001-5760-5172

1. Dirección de Investigación en Diversidad Biológica Terrestre Amazónica (DBIO), Instituto de Investigaciones de la Amazonía Peruana, Iquitos, Perú; jvasquez@iiap.gob.pe (Correspondencia*),norellis.jeda03@gmail.com

2. Facultad de Agronomía, Universidad Nacional de la Amazonía Peruana, Iquitos, Perú; julio.pinedo@unapiquitos.edu.pe

3. Facultad de Biología, Universidad Nacional de la Amazonía Peruana, Iquitos, Perú; alberto.garcia@unapiquitos.edu.pe

4. Facultad de Forestales, Universidad Nacional de la Amazonía Peruana, Iquitos, Perú; heiter.valderrama@unapiquitos.edu.pe

5. Museo de Historia Natural, Universidad Nacional Mayor de San Marcos, Lima, Perú; macrodontia@hotmail.com

Recibido 12-I-2020. Corregido 14-III-2021. Aceptado 18-III-2021.

\author{
ABSTRACT \\ Feeding, oviposition behavior, life cycle, and natural enemies \\ of Hamadryas Feronia (Nymphalidae) in the Peruvian Amazon
}

Introduction: Hamadryas feronia feronia, which mimics the bark of trees and often makes attention-grabbing sounds when flying, has good potential for bio-business (export, handicrafts and tourist breeding centers). However, its biological aspects are still unknown, which hinder its captive breeding. Objectives: To determine the biological aspects of feeding, oviposition behavior, life cycle and natural enemies of Hamadryas feronia feronia L. in San Rafael-Indiana, Loreto, Peru. Methods: Sampling was conducted from January 2018 to December 2019 in the community of San Rafael, Amazon River. Adults were observed during the day, their food plants, oviposition behavior, biological cycle and natural enemies were recorded. The life cycle was evaluated in the laboratory, using 20 eggs recently deposited on the leaves of their host plant. Results: The larvae of H. feronia feronia feed on the leaves of Dalechampia juruana and the adults feed on the bark sap of Cedrela odorata, Spondias mombin, Uncaria guianensis and the fermented fruits of Syzygium malaccense and Pouteria caimito. Adults fly on sunny days, males emit a loud sound when flying. Females prior to oviposition flit irregularly around their host plant between 8.00 and $14.00 \mathrm{~h}$ and deposit their eggs on the upper and underside of leaves in isolation, most frequently on the underside. The duration of the cycle from egg to adult was 28.24 days. The average egg period was $3.75 \pm 0.40$ days. The larvae passed through five larval instars: the first instar $3.21 \pm 1.03$ days, the second $2.78 \pm 0.73$ days, the third $2.67 \pm 0.77$ days, the fourth $3.22 \pm 0.81$ days, and the fifth $4.61 \pm$ 0.70 days. The prepupal period lasted $1.33 \pm 0.49$ days and the pupal period $6.67 \pm 0.80$ days; the adults hatched between 10:00 to 11:00 h. Adult males lived on average $31.80 \pm 3.29$ days, the female $42.00 \pm 2.14$ days and their eggs were parasitized by a microhymenopteran (Scelionidae). Conclusions: This study allowed to know 


\begin{abstract}
the biological aspects of $H$. feronia feronia identifying its food plants of both larvae and adults, its oviposition behavior, as well as it has been determined that it has a relatively short biological cycle with a period of less than one month and its eggs are consumed by a small Hymenoptera that can hinder its production. This work provides necessary information to develop the breeding of $H$. feronia feronia, oriented to its conservation, environmental education and biotrade (tourism and handicrafts) in the Peruvian Amazon.
\end{abstract}

Key words: food plant; oviposition; eggs; larva; life cycle; parasitoid.

La mariposa Hamadryas feronia (Linnaeus, 1758) ocurre en el Norte, Centro y Sur de América, desde Estados Unidos hasta Argentina (Thomas, 1991; Yack, Otero, Dawson, Surlykke, \& Fullard, 2000; Monge-Nájera \& Gómez, 2003; Brown et al., 2007; Glassberg, Jeffrey, \& Quinn, 2008; Marini-Filho \& Benson, 2010; Roman, Garlet, \& Costa, 2010; Prado, Pozo, Valdez-Moreno, \& Hebert, 2011; Avigliano \& Schenone, 2015; Casas-Pinilla, Mahecha, Dumar, \& Ríos-Málaver, 2017; Palacios-Mayoral, Palacios-Mosquera, \& JiménezOrtega, 2018; Maes, 2020). La subespecie $H$. feronia feronia (Linnaeus, 1758) se distribuye desde Ecuador, Brasil, Paraguay, Perú y Argentina (Scott, 1992; Murillo-Hiller, 2012; Thiele, Milcharek, dos Santos, \& Kaminski, 2014; Avigliano \& Schenone, 2015; Silva et al., 2015). Las larvas de H. feronia feronia son oligófagas y se alimentan de las hojas de varias especies del género Dalechampia en el Neotrópico (Monge-Nájera \& Gómez, 2003; Beccaloni, Viloria, Hall, \& Robinson, 2008). Los adultos consumen el jugo de frutos y la savia de la corteza de los árboles (MongeNájera et al., 1998; Junior \& Diniz, 2015; Silva et al., 2015; González-Valdivia et al., 2016; Araujo, Martins, Duarte, \& Azevedo, 2020; Porath \& Aranda, 2020). La mariposa H. feronia es conocida como la mariposa tronadora o sonora en Costa Rica, por el extraordinario y llamativo sonido que producen, lo cual fue una fuente de inspiración científica que dio inicio a diversas investigaciones, orientadas a su diversidad, la producción de sonido, su morfología distribución espacial, territorialidad, mimetismo y sus plantas alimenticias (Sargent \& Keiper, 1969; Monge-Nájera \& Hernández, 1991; Monge-Nájera et al., 1998; Yack et al., 2000;
Monge-Nájera \& Gómez, 2003; Beccaloni et al., 2008; González-Valdivia et al., 2016; Casas-Pinilla et al., 2017; Palacios-Mayoral et al., 2018; Porath \& Aranda, 2020).

Estas mariposas son consideradas como una de las especies de gran atracción turística en el criadero "Morphosapi" ubicado en la comunidad de San Rafael en la Amazonía del Perú, debido al sonido que emiten y al mimetismo que las hace pasar desapercibidas en la corteza de los árboles. Además, son un potencial para los bionegocios, ya que cada individuo (A1) se comercializa a un precio de 2 euros; sin embargo, la principal problemática que obstaculiza su crianza masiva y sostenible en cautiverio es que no existe información sobre sus aspectos biológicos, adaptación reproductiva bajo condiciones de cautiverio, ciclo biológico, sus plantas hospederas y sus enemigos naturales en la amazonia del Perú.

Los objetivos de este estudio fueron determinar los aspectos biológicos de alimentación, comportamiento de oviposición, ciclo de vida y sus enemigos naturales de $H$. feronia feronia, con el propósito de desarrollar su manejo sostenible, orientado a su conservación, la educación ambiental y los bionegocios (turismo y artesanía) en la Amazonia peruana.

\section{MATERIALES Y MÉTODOS}

Área de estudio: El estudio se desarrolló, desde enero 2017 a diciembre 2019, en la Comunidad Campesina de San Rafael, cuyas coordenadas son: $3^{\circ} 33^{\prime} 40.21^{\prime \prime}$ S \& $73^{\circ} 07^{\prime} 07.17^{\prime} \mathrm{W}$, presenta un clima cálido y húmedo, con temperatura media de $26^{\circ} \mathrm{C}$ y precipitación promedio anual de $2900 \mathrm{~mm}$, donde el periodo que transcurre entre enero hasta 
febrero es la época de mayor lluvia. La zona cuenta con un bosque secundario, presencia de las mariposas $H$. feronia feronia, mariposarios experimentales y sus plantas hospederas del género Dalechampia, desarrolladas de forma natural y también sembradas.

Evaluación de las plantas alimenticias y comportamiento reproductivo: En las áreas de estudio, los adultos fueron observados desde las $6 \mathrm{~h}$ hasta las $17 \mathrm{~h}$. Se registraron a los adultos durante su alimentación, la hora de vuelo y proceso de oviposición sobre su planta hospedera. Las plantas alimenticias detectadas fueron recolectadas y herborizadas para su determinación taxonómica, así mismo, las larvas eclosionadas fueron criadas con su planta de origen hasta obtener los adultos para su identificación taxonómica. Los adultos obtenidos de la evaluación del ciclo biológico fueron rotulados en una de las alas y liberados en un mariposario de $80 \mathrm{~m}^{2}$ X $6 \mathrm{~m}$ de altura, en cuyo interior fueron sembradas sus plantas hospederas para observar su comportamiento reproductivo (cortejo, copula, oviposición y longevidad) estas mariposas fueron alimentadas con rodajas del fruto maduro de Musa paradisiaca (plátano) macerado en jugo de Saccharum officinarum L. (caña de azúcar).

Identificación taxonómica: La determinación taxonómica de las mariposas se realizó a través de claves taxonómicas y por comparación con ejemplares de la colección del Museo de Historia Natural de la Universidad Nacional Mayor de San Marcos (MHMSM). Los ejemplares se encuentran conservados en el laboratorio de entomología del Instituto de Investigaciones de la Amazonia Peruana (IIAP). Las plantas alimenticias de las larvas y los adultos fueron determinadas taxonómicamente, a través de claves taxonómicas (Brako \& Zarucci, 1993; Vasquez \& Rojas, 2016) y fueron comparadas con exsicatas o voucher del Herbarium Amazonense (AMAZ) de la Universidad Nacional de la Amazonia Peruana (UNAP).
Evaluación de su ciclo biológico: El ciclo biológico fue registrado en el laboratorio del zoocriadero "Morphosapi" a una temperatura entre 26 a $29^{\circ} \mathrm{C}$ y una humedad relativa de 80 $\%$, a partir de los huevos recolectados desde el campo o el mariposario. Después de que la hembra terminó el proceso de oviposición, se retiró la hoja que contenía los huevos y se colocaron en envases plásticos para su transporte al laboratorio. En este lugar se confinaron en una placa petri y se controló el periodo en el que emergieron las larvas, registrándose los días que duraba el proceso. Luego de la emergencia, las larvas fueron individualizadas en envases plástico de $1 \mathrm{~L}$ trasparente con tapa, en cuyo interior se colocaron pequeños floreros con hojas de su planta hospedera sumergida en agua, todo esto para evitar su marchitamiento y que la larva se desarrollase sin dificultad. La limpieza de las unidades de cría inicialmente se realizó cada dos días, pero cuando las larvas empezaron a desarrollarse, se les alimentaba diariamente, eliminando las excretas y el alimento sobrante, evitando la proliferación de entomopatógenos. El número de estadíos larvarios fue controlado a través del proceso de muda y el indicador fue la presencia de la cápsula cefálica, que fue medida con una regla micrométrica. El periodo de los estadíos larvales se registró luego de cada muda, las larvas fueron medidas cada vez que ellas cambiaban de estadío a través de un estereoscopio con regla graduada en milímetros $(\mathrm{ml})$ y a medida que incrementaba su tamaño se utilizó un vernier. Para registrar el periodo de la prepupa, observamos a las larvas que no se alimentaban e iniciaban una decoloración. La fase de pupa fue caracterizada y medida con el vernier, controlando posteriormente su periodo hasta la emergencia de los adultos.

Enemigos naturales: Para evaluar sus enemigos naturales se recolectaron muestras de huevos, larvas y pupas del mariposario y de sus plantas hospederas. Los huevos fueron acondicionados en placas petri, las larvas y pupas en envases herméticos de plástico de 1 
L en condiciones de laboratorio y se evaluó la presencia de sus enemigos naturales.

\section{RESULTADOS}

\section{Plantas alimenticias y comportamiento:} Los adultos de $H$. feronia feronia fueron observados alimentándose de la savia (resina) de la corteza de algunos árboles como Cedrela odorata "cedro", Spondias mombin "ubos" y de la liana Uncaria guianensis "uña de gato" y de los frutos fermentados de Syzygium malaccense "pomarrosa" y Pouteria caimito "caimito". Además, se observaron a los machos alimentándose de excreta de "perro" Canis familiaris. Los adultos se posan con las alas abiertas en los árboles circundantes a su planta hospedera, donde su coloración les permite pasar desapercibidos sobre la corteza de los árboles. Los adultos fueron muy activos en días soleados, los machos al volar emiten un sonido como un estruendo fuerte y las hembras realizaron revoloteos de forma irregular alrededor de su planta hospedera (Dalechampia juruana) donde depositaron sus huevos entre las 8.00 y las $14.00 \mathrm{~h}$ principalmente en el haz y envés de las hojas de forma aislada observándose la mayor frecuencia de oviposición en el envés $(\mathrm{N}=85)$. Sus larvas se alimentaron de las hojas maduras.

Ciclo biológico. Huevos (Fig. 1A): De color blanco perlado, de forma elíptica con la superficie apical y basal plana provista de estrías verticales. Duración promedio: 3.75 \pm 0.40 días. Diámetro promedio: $1.50 \pm 0.07$ $(\mathrm{N}=20)$.

Estadio I (Fig. 1B): Desde que nace se alimenta de la hoja y construye con su seda y sus pequeñas excretas una prolongación en su borde donde se esconde. Desde allí sale a alimentarse y luego regresa a su escondite donde queda inmóvil pasando desapercibida (camuflado) y protegiéndose de sus depredadores. Es de color hialino con cabeza negra, presenta en el cuerpo unas pequeñas protuberancias de color blanquecino ubicadas en el dorso a manera de líneas paralelas que van desde el tórax hasta el final del abdomen, a medida que pasan los días se torna de color marrón y las protuberancias de color blanco. El ancho medio de la cápsula de la cabeza fue $0.80 \pm 0.00 \mathrm{~mm}$, la longitud media de la larva: $2.51 \pm 0.02 \mathrm{~mm}$ y la duración media: $3.21 \pm 1.03$ días $(\mathrm{N}=19)$.

Estadio II (Fig. 1C): De color marrón oscuro y la cabeza negra, en donde aparecen dos cuernos truncados con minúsculas setas. El cuerpo está cubierto de setas, en su mayoría son negras, algunas combinadas con tonos blancos, las de posición dorsal son largas en forma de espolones provistas de espinas. Las setas más desarrolladas se encuentran en el tórax y los segmentos abdominales A10 y A11. El ancho medio de la cápsula de la cabeza fue $1.19 \pm$ $0.03 \mathrm{~mm}$, la longitud media de la larva: 4.55 $\pm 0.16 \mathrm{~mm}$ y la duración media: $2.78 \pm 0.73$ $(\mathrm{N}=18)$.

Estadio III (Fig. 1D): De color negro con puntos blancos en el cuerpo. La zona ventral del cuerpo es de color marrón que se extiende en toda la longitud del cuerpo como una banda que ocupa la mitad de la zona pleural. Los cuernos son prolongados y curvados hacia atrás con presencia de setas y un ápice en forma de maza, la cabeza se mantiene de color negro. Las setas del cuerpo son muy visibles con diversas espinas y son más desarrolladas en el tórax y los segmentos abdominales A10 y A11. En este estadío la larva abandona su escondite y se refugia debajo de la hoja. El ancho medio de la cápsula de la cabeza fue $1.71 \pm 0.02 \mathrm{~mm}$, la longitud media de la larva: $8.86 \pm 0.23 \mathrm{~mm}$ y la duración media: $2.67 \pm 0.77(\mathrm{~N}=18)$.

Estadio IV (Fig. 1E): De color marrón moteado con una banda ancha en el dorso de tono más claro, la zona pleural presenta manchas rosadas, los cuernos son similares al estadío anterior, pero de mayor tamaño. La cabeza se mantiene de color negro y presenta pequeñas espinas, se conservan las setas del cuerpo y se observan grupos de setas dorsales gruesas y más desarrolladas cubiertas de espinas. Dos pares de estas setas están ubicadas en 

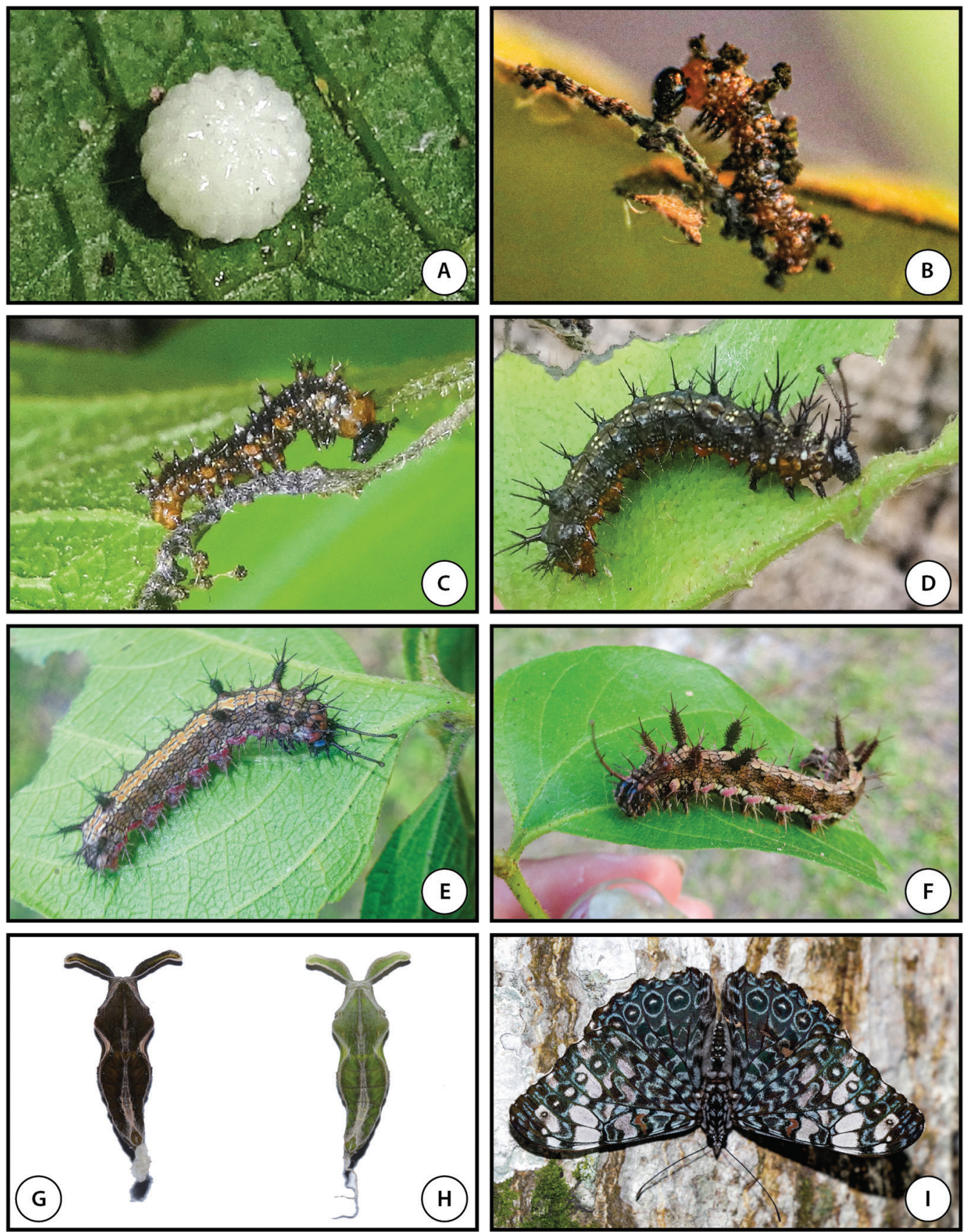

Fig. 1. A. Huevo de H. feronia feronia sobre hoja de Dalechampia juruana, B. Primer estadío, C. Segundo estadío, D. Tercer estadío, E. Cuarto estadío, F. Quinto estadío, G. Pupa marrón, H. Pupa Verde, I. Adulto macho de H. feronia feronia. Fig. 1. A. Egg of H. feronia feronia on leaf of Dalechampia juruana, B. 1st instar, C. 2nd instar, D. 3rd instar, E. 4th instar, F. 5th instar, G. Pupae Brown, H. Pupae green, I. Male adult of H. feronia feronia. 
la región torácica en los segmentos $\mathrm{T} 2, \mathrm{~T} 3 \mathrm{y}$ cinco setas en la región abdominal; el primer par se ubica en el segmento A2, una en el centro del segmento A10 y dos en el segmento A11. La larva se refugia en el envés de las hojas. El ancho medio de la cápsula de la cabeza fue $2.51 \pm 0.05 \mathrm{~mm}$, la longitud media de la larva: $17.67 \pm 1.64 \mathrm{~mm}$ y su duración media: $3.22 \pm 0.81(\mathrm{~N}=18)$.

Estadio V (Fig. 1F): La larva mantiene las características del estadío anterior, su cuerno se torna de color rosado pálido. La cabeza presenta manchas irregulares y dos bandas paralelas del mismo color de la antena. Cápsula de la cabeza: anchura: $3.90 \pm 0.03 \mathrm{~mm}$. Longitud media de la larva: $22.56 \pm 1.74 \mathrm{~mm}$. Duración media: $4.61 \pm 0.70(\mathrm{~N}=18)$.

La prepupa: De color marrón pálido, el cuerpo se motea y las manchas de color rosado en la zona pleural se hacen visiblemente más pálidas, al final de su periodo pierde su color original, tornándose de color verde pálido. La larva se adhiere al sustrato y deja de alimentarse. La longitud media de la prepupa: $27.31 \pm$ $3.26 \mathrm{~mm}$ y su duración media fue $1.33 \pm 0.49$ días $(\mathrm{N}=18)$.

Pupa: Las pupas habitualmente son de color verde olivo (Fig. 1G) y marrón (Fig. 1H). El borde dorsal del tórax y del abdomen está definido por una banda de color blanquecina de forma romboide. El vértice presenta dos apéndices foliares a manera de cuernos aplanados. Se observa una línea que recorre el centro del dorso desde el tórax hasta el final del abdomen. La longitud media de la pupa, desde el vértice hasta el final del abdomen fue $27.86 \pm 1.20$ $\mathrm{mm}$, su longitud media de los apéndices foliares: $5.83 \pm 1.08 \mathrm{~mm}$ y su duración media: 6.67 \pm 0.80 días $(\mathrm{N}=18)$.

Los adultos: Nacen entre las 10:00 y las 11:00 h. La longevidad promedio del macho (Fig. 1I) fue $31.80 \pm 3.29$ días $(\mathrm{N}=10)$ y de la hembra de $42.00 \pm 2.14$ días $(\mathrm{N}=8)$. Es posible que los adultos sean más longevos debido a que la mayoría de los adultos muertos presentaban las alas magulladas por el roce con las mallas durante el vuelo, imposibilitando su desplazamiento hacia el alimento. Los machos al segundo día cortejan a las hembras en horas del mediodía en días soleados, suelen perseguir a las hembras, ellas se posan en los troncos de los árboles el macho se posa detrás de ella y se acerca lentamente hacia la hembra, sin embargo, se retira volando y el macho lo persigue con insistencia y cuando la hembra esta apta acepta la copula que ocurrió entre el cuarto y el sexto día. Se observó una sola copula por pareja $(\mathrm{N}=$ 5) y la oviposición ocurrió al séptimo día.

Enemigos Naturales: Se identificó un enemigo natural que parasita los huevos de $H$. feronia feronia, se trata de un microhimenóptero (Scelionidae), de color negro con alas transparentes de $0.2 \mathrm{~mm}$ de longitud, la antena de la hembra es acodadas y su abdomen ensanchado; en cambio de los machos sus antenas son filiformes y su abdomen delgado. Los huevos parasitados fueron encontrados tanto en condiciones de cautiverio (mariposario), en las plantas sembradas, así como en el medio natural cuando eran colectados a partir del tercer día de su postura.

\section{DISCUSIÓN}

Se ha identificado a D. juruana como única planta hospedera de Hamadryas feronia feronia, esta especie tiene hábito oligófaga, es decir se alimenta de varias especies del género Dalechampia, tal como lo reportan Monge-Nájera y Gómez, (2003) quienes precisan que las hembras de cada especie del género Hamadryas utilizan una especie diferente de planta hospedera del género Dalechampia. Del mismo modo Beccaloni et al. (2008) reportan como plantas hospederas de $H$. feronia feronia a Dalechampia scandens en Trinidad y Venezuela, Dalechampia stenosepala en Brasil y Uruguay, Dalechampia tiliifolia en Venezuela y Dalechampia triphylla en Argentina, Brasil y Sur América. Se ha observado que los adultos tienen hábito frugívoro y se alimentan además 
de savia de la corteza de los árboles, resultados similares reportan Monge-Nájera et al. (1998), Junior y Diniz (2015), Silva et al. (2015), González-Valdivia et al. (2016), Araujo et al. (2020), Porath y Aranda (2020), los machos además suelen alimentarse de excretas de animales, éste proceder es común en ninfálidos de la familia Charaxinae y Biblidinae, tal es el caso de machos de Panacea prola amazónica los que fueron observados succionando excretas de animales (Mulanovic, 2007; Vasquez, Lamas, Couturier, \& Mejia, 2012). Los adultos de $H$. feronia feronia se caracterizan por posarse en la corteza de los árboles con la cabeza hacia abajo y con las alas abiertas pasando desapercibidos (DeVries, Murray, \& Lande, 1997; Monge-Nájera et al., 1998; Contreras \& Contreras, 2010). Los adultos de ambos sexos descansan en los árboles circundantes a su planta hospedera, similar conducta posee Hamadryas februa (Young, 1974) y son muy activos en días soleados, este comportamiento es común en las mariposas amazónicas (Mulanovic, 2007). H. feronia utiliza el sonido para el cortejo, para defender su territorio y además se le atribuye como un mecanismo de defensa cuando se acercan posibles depredadores (Monge-Nájera et al., 1998; Marini-Filho \& Benson, 2010), sin embargo, el aparato de sonido no solo es exclusivo de los machos, también existe en ambos sexos, siendo ligeramente mayor en los machos (Monge-Nájera et al., 1998). En nuestro caso observamos emitir sonidos solo a los machos durante el vuelo y el cortejo. La estructura encargada de emitir sonidos (posiblemente, percusión por movimiento de las alas) se debe a la base ensanchada de la vena subcostal presente exclusivamente en Hamadryas que posee una estructura serpentina en su interior y probablemente actúa como caja de resonancia (Monge-Nájera \& Hernández, 1991; MongeNájera et al., 1998). En Venezuela la actividad de $H$. feronia comienza entre las 09:30 h y las 10:00 h, con picos antes de las 12:00 h, disminuyendo su actividad cuando alcanza las horas más calurosas del día (Contreras \& Contreras, 2010). En Costa Rica las horas activas de $H$. feronia ocurren entre las 13:00 h y las 15:00 h y son más frecuentes en la temporada de lluvias (Monge-Nájera \& Hernández, 1991; Monge-Nájera et al., 1998). Estas aseveraciones son parecidas a nuestras observaciones en los adultos de $H$. feronia feronia. Con respecto a su comportamiento de oviposición los resultados de este trabajo muestran que las hembras de $H$. feronia feronia revolotean alrededor de su planta hospedera, colocan sus huevos en el haz y el envés de las hojas de forma aislada con mayor frecuencia en el envés y descansan en los árboles circundantes. En este sentido, similar comportamiento fue observado en $H$. februa por Young (1974) y en Hamadryas epinome por Ribeiro, Silva, Carneiro, Martins, y Hendrik (2012). Este comportamiento difiriere de Hamadryas fornax fonax que realiza su oviposición en el haz de la hoja en forma gregaria, uno encima del otro y sus larvas emergen en sentido lateral (Garcia, Ribeiro, Silva, Martins, \& Hendrik, 2015). Respecto al tamaño de los huevos, destaca que existe una variación notable. Los huevos de $H$. feronia feronia son relativamente más grandes que los huevos de $H$. epinome, $H$. fornax fornax y de $H$. februa (Young, 1974; Ribeiro et al., 2012; Garcia et al., 2015). Las larvas pasan por cinco estadios al igual que $H$. epinome y $H$. fornax fornax, de H. februa, Hamadryas glauconome (Young, 1974; Ribeiro et al., 2012; Garcia et al., 2015).

La larva del primer estadio no presenta cuernos en la cabeza, estas estructuras aparecen a partir del segundo estadío y su longitud inicial es muy similar a la de H. februa (Young, 1974). Las larvas en el quinto estadio son muy parecidos en forma y tamaño a $H$. epinome, H. fornax fornax, H. februa y H. glauconome, presenta cuernos largos y setas ramificadas en todo el cuerpo, diferenciándose morfológicamente de las otras Hamadryas por las setas ramificadas y gruesas sobresalientes ubicadas en los segmentos T2, T3, A2, A10 y A11, con excepción a la de $H$. guatemalena que es muy similar (Young, 1974; Ribeiro et al., 2012; Nall, 2014; Garcia et al., 2015). La pupa es alargada, de color verde o marrón en la cabeza, presenta extensiones laminadas a manera de cuernos y se asemeja a la pupa de H. epinome, 
H. fornax fornax, H. februa, H. glauconome y H. guatemalena (Young, 1974; Ribeiro et al., 2012; Nall, 2014; Garcia et al., 2015). La coloración verde o marrón adquirida al inicio de la pupa se mantiene durante este periodo y un día antes de la emergencia se oscurece. En cambio, las pupas recién formadas de $H$. epinome y $H$. fornax fornax son de color verde claro ventralmente, verde oliva en el dorso, luego de un período mínimo de veinticuatro horas cambian a un tono marrón claro ventralmente y un tono marrón verdoso en el dorso (Ribeiro et al., 2012; Garcia et al., 2015).

La duración promedio del ciclo total de $H$. feronia feronia desde huevo hasta la emergencia del adulto es relativamente más corto que el ciclo de H. februa, H. fornax fornax y H. epinome (Young, 1974; Ribeiro et al., 2012; Garcia et al., 2015). El periodo de los ciclos biológicos depende mucho de la variación de la temperatura (Hurtado, 2009). La longevidad promedio de los adultos fue menor a lo encontrado por Monge-Nájera et al. (1998) para H. feronia en Costa Rica quienes indican una longevidad que podría superar los 14 meses, debido a su alimentación con líquidos más nutritivos que se encuentran en la savia fermentada y frutas descompuestas en condiciones silvestres. Sin embargo, si durante el vuelo no lastimaran sus alas, lo que les impidió volar hacia el alimento, es muy probable que la longevidad de los adultos hubiera sido más prolongada.

Con relación a los enemigos naturales, se encontró un pequeño insecto que parasita los huevos de $H$. feronia feronia cuyas características son muy parecidas al parasitoide de los huevos de Panacea prola amazonica, Morpho helenor theodoros y Mechanitis polymnia (Vasquez et al., 2012; Ruíz, Vásquez, Zárate, \& Pinedo, 2015).

Declaración de ética: Todos los autores declaran que están de acuerdo con esta publicación y que han hecho aportes que justifican su autoría; no existe conflicto de interés de ningún tipo; y que cumplieron con todos los requisitos y procedimientos éticos y legales adecuados.
Todas las fuentes de financiamiento se detallan con claridad en la sección de agradecimientos. El respectivo documento legal firmado se encuentra en los archivos de la revista.

\section{AGRADECIMIENTOS}

Este trabajo fue apoyado por el "Programa Nacional de Innovación para la Competitividad y Productividad, Innóvate-Perú" a través del subproyecto "Modelos tecnológicos de crianza de 10 especies de mariposas diurnas para su aprovechamiento en bionegocios en la Región Loreto", conducido por la Dirección de Investigación en Biodiversidad Amazónica Terrestre del Instituto de Investigaciones de la Amazonía Peruana en cooperación con la Universidad Nacional de la Amazonia Peruana. Los autores agradecen a Pedro Vela García por su apoyo durante los muestreos y evaluaciones en el campo y laboratorio. A Alesandro Tupia por las tomas fotografías de las mariposas, a Manuel Martin Brañas y Juan José Bellido Collahuacho por la revisión del manuscrito.

\section{RESUMEN}

Introducción: Hamadryas feronia feronia, se mimetiza sobre las cortezas de los árboles y suele emitir sonidos al volar que llaman la atención; posee un buen potencial para los bionegocios (exportación, elaboración de artesanías y centros turísticos de crianza). Sin embargo, aún se desconocen sus aspectos biológicos que obstaculizan su crianza en cautiverio. Objetivos: Determinar los aspectos biológicos de alimentación, comportamiento de oviposición, ciclo de vida y los enemigos naturales de Hamadryas feronia feronia L. en San Rafael-Indiana, Loreto, Perú. Métodos: Los muestreos fueron realizados desde enero 2018 a diciembre 2019 en la comunidad de San Rafael, río Amazonas. Los adultos fueron observados durante el día, se registraron sus plantas alimenticias, su comportamiento de oviposición, su ciclo biológico y sus enemigos naturales. El ciclo de vida fue evaluado en el laboratorio, utilizando 20 huevos recientemente depositados en las hojas de su planta hospedera. Resultados: Las larvas de $H$. feronia feronia se alimentan de las hojas de Dalechampia juruana y los adultos se alimentan de la savia de la corteza de los árboles de Cedrela odorata, Spondias mombin, Uncaria guianensis y de los frutos fermentados de Syzygium malaccense y Pouteria caimito. Los adultos vuelan en días soleados, los machos emiten un fuerte sonido al volar. Las hembras previo a la oviposición revolotean de forma 
irregular alrededor de su planta hospedera entre las $8.00 \mathrm{y}$ las $14.00 \mathrm{~h}$ y depositan sus huevos en el haz y envés de las hojas de forma aislada con mayor frecuencia en el envés $(\mathrm{N}=85)$. La duración del ciclo, desde huevo hasta adulto fue de 28.24 días. El periodo promedio del huevo fue 3.75 \pm 0.40 días. La larva pasa por cinco estadíos larvales: el primero duró $3.21 \pm 1.03$ días, el segundo $2.78 \pm 0.73$ días, el tercero $2.67 \pm 0.77$ días, el cuarto $3.22 \pm 0.81$ días, y el quinto $4.61 \pm 0.70$ días. El periodo de la prepupa duró 1.33 \pm 0.49 días y el de pupa $6.67 \pm 0.80$ días; los adultos nacieron entre las 10:00 y 11:00 h. Los machos adultos viven en promedio $31.80 \pm 3.29$ días, la hembra $42.00 \pm 2.14$ días $\mathrm{y}$ sus huevos son parasitados por un microhimenóptero (Scelionidae). Conclusiones: Este estudio permitió conocer los aspectos biológicos de $H$. feronia feronia identificando sus plantas alimenticias tanto de las larvas como de los adultos, su comportamiento de oviposición, así mismo se ha determinado que tiene un ciclo biológico relativamente corto con un periodo menor de un mes y sus huevos son consumidos por un pequeño Himenóptero que puede obstaculizar su producción. Este trabajo brinda información necesaria para desarrollar la crianza de $H$. feronia feronia, orientado a su conservación, la educación ambiental y los bionegocios (turismo y artesanía) en la Amazonia peruana.

Palabras clave: planta alimenticia; oviposición; huevos; larva; ciclo de vida; parasitoide.

\section{REFERENCIAS}

Araujo, E.C., Martins, L.P., Duarte, M., \& Azevedo, G.G. (2020). Temporal distribution of fruit-feeding butterflies (Lepidoptera, nymphalidae) in the eastern extreme of the Amazon region. Acta Amazonica, 50(1), 12-23. DOI: 10.1590/1809-4392201901261

Avigliano, E., \& Schenone, N.F. (2015). Nymphalidae, papilionidae and pieridae (Insecta: Lepidoptera: Rhopalocera) from the acaraguá river basin, Misiones, Argentina. Entomotropica, 30(9), 84-91.

Beccaloni, G.W., Viloria, A.L., Hall, S.K., \& Robinson, G.S. (2008). Catalogue of the hostplants of the Neotropical butterflies. Zaragoza, España: Monografías Tercer Milenio.

Brako, L., \& Zarucci, J.L. (1993). Catalogue of the flowering plants and gymnosperms of Peru. Missouri, EUA: Missouri Botanical Garden.

Brown, K.S., Lucci, A.V., Wahlberg, N., Von Schoultz, B., Saura, A.O., \& Saura, A. (2007). Chromosomal evolution in the South American Nymphalidae. Hereditas, 144, 137-148. DOI: 10.1111/j.2007.0018-0661.02015.x

Casas-Pinilla, L.C., Mahecha, O., Dumar, J.C., \& RíosMálaver, I.C. (2017). Diversidad de mariposas en un paisaje de bosque seco tropical, en la Mesa de los Santos, Santander, Colombia (Lepidoptera:
Papilionoidea). SHILAP Revista de Lepidopterología, 45(177), 83-108.

Contreras, A., \& Contreras, J. (2010). Presencia del género Hamadryas Hübner, 1825 (Lepidoptera: Nymphalidae: Biblidinae), en la Ecorregión del Ñeembucú y en el resto del Paraguay Oriental. Azariana, 1(21), 225-242.

DeVries, P.J., Murray, D., \& Lande, R. (1997). Species diversity in vertical, horizontal, and temporal dimensions of a fruit-feeding butterfly community in an Ecuadorian rainforest. Biological Journal of the Linnean Society, 62, 343-364. DOI: 10.1006/bijl.1997.0155

Garcia, L.M., Ribeiro, L.A., Silva, F.M., Martins, M., \& Hendrik, O.H. (2015). Immature stages of Hamadryas fornax fornax (Hübner) (Lepidoptera: Nymphalidae: Biblidinae). Revista Brasileira de Entomologia, 59, 301-306. DOI: 10.1016/j.rbe.2015.08.002

Glassberg, E.C., Jeffrey, K., \& Quinn, M. (2008). Butterflies of Santa Ana National Wildlife Refuge. Texas, EUA: Service, U.S. Fish \& Wildlife.

González-Valdivia, N.A., Pozo, C., Ochoa-Gaona, S., Ferguson, B.G., Cambranis, E., Lara, O., PérezHernández, I., Ponce-Mendoza, A., \& Kampichler, C. (2016). Nymphalidae frugívoras (Lepidoptera: Papilionoidea) asociadas a un ecomosaico agropecuario y de bosque tropical lluvioso en un paisaje del sureste de México. Revista Mexicana de Biodiversidad, 87(2), 451-464. DOI: 10.1016/j.rmb.2016.04.003

Hurtado, L. (2009). Descripción del ciclo biológico de Utetheisa ornatrix venusta (Dalm) (Lepidoptera: Arctiidae) con una dieta artificial. Centro Agrícola, 36(4), 79-84.

Junior, G.B., \& Diniz, I.R. (2015). Temporal dynamics of fruit-feeding butterflies (Lepidoptera: Nymphalidae) in two habitats in a seasonal Brazilian environment. Florida Entomologist, 98(4), 1207-1216. DOI: 10.1653/024.098.0430

Maes, J.M. (2020). Distribucion geografica de Myscelia ethusa pattenia BUTLER \& DRUCE, 1972 (Lepidoptera: Nymphalidae) en Nicaragua. Revista Nicaraguense de Entomología, 195, 1-30.

Marini-Filho, O.J., \& Benson, W.W. (2010). Use of sound and aerial chases in sexual recognition in Neotropical Hamadryas butterflies (Nymphalidae). Journal of Research on the Lepidoptera, 42, 5-12.

Monge-Nájera, J., \& Gómez, P. (2003). Las mariposas tronadoras (Hamadryas spp.) y su distribución geográfica en Costa Rica. Biocenosis, 17(1), 22-26.

Monge-Nájera, J., \& Hernández, F. (1991). A morphological search for the sound mechanism of Hamadryas butterflies (Lepidoptera: Nymphalidae). Journal of Research on the Lepidoptera, 30(3-4), 196-208. 
Monge-Nájera, J., Hernández, F., González, M.I., Soley, J., Araya, J., \& Zolla, S. (1998). Spatial distribution, territoriality and sound production by tropical cryptic butterflies (Hamadryas, Lepidoptera: Nymphalidae): Implications for the "industrial melanism" debate. Revista de Biología Tropical, 46(2), 297-330.

Mulanovic, A. (2007). Guía para el manejo sustentable de las mariposas del Perú. Lima, Perú: Instituto de Investigaciones de la Amazonia Peruana.

Murillo-Hiller, L.R. (2012). Phylogenetic Analysis of the Subtribe Ageroniina with Special Emphasis on Hamadryas (Lepidoptera, Nymphalidae) with an Identification Key to the Species of Hamadryas. International Scholarly Research Network Zoology, 2012, 1-17. DOI: 10.5402/2012/635096

Nall, B. (2014). Rearing Hamadryas (Nymphalidae: Biblidinae: Ageronini) in South Texas: life histories of three species. News of the Lepidopterists Society, 56(3), 103-112. DOI: 10.7748/ns.3.46.52.s64

Palacios-Mayoral, V.D., Palacios-Mosquera, L., \& Jiménez-Ortega, A.M. (2018). Diversidad de mariposas diurnas (Lepidoptera: Papilionoidea) asociadas con tres hábitats en el corregimiento de Pacurita, municipio de Quibdó, Chocó, Colombia. Revista de La Academia Colombiana de Ciencias Exactas, Físicas y Naturales, 42(164), 237-245. DOI: 10.18257/ raccefyn. 607

Porath, I.A.T., \& Aranda, R. (2020). Frugivorous butterflies (Lepidoptera: Nymphalidae) as a habitat quality indicator in Cerrado urban fragment. EntomoBrasilis, 13(July), e904. DOI: 10.12741/ebrasilis.v13.e904

Prado, B.R., Pozo, C., Valdez-Moreno, M., \& Hebert, P.D.N. (2011). Beyond the colours: Discovering hidden diversity in the nymphalidae of the Yucatan Peninsula in Mexico through DNA barcoding. PLoS ONE, 6(11), 1-11. DOI: 10.1371/journal. pone.0027776

Ribeiro, L.A., Silva, F.M., Carneiro, E., Martins, M., \& Hendrik, O.H. (2012). Immature Stages of the Neotropical Cracker Butterfly, Hamadryas epinome. Journal of Insect Science, 12(74), 1-12. DOI: 10.1673/031.012.7401

Roman, M., Garlet, J., \& Costa, E.C. (2010). Levantamento populacional e comportamento de voo de borboletas (Lepidoptera) em um remanescente florestal em são sepé, rs. Ciencia Florestal, 20(2), 283-294. DOI: $10.5902 / 198050981852$

Ruíz, E., Vásquez, J., Zárate, R., \& Pinedo, J. (2015). Aspectos Biológicos de Morpho helenor theodorus (Fruhstorfer) y Mechanitis polymnia (Linnaeus), en la Amazonía baja del Perú. Folia Amazónica, 24(1), 45. DOI: $10.24841 /$ fa.v24i1.54

Sargent, T.D., \& Keiper, R.R. (1969). Behavioral adaptations of cryptic moths. I. Preliminary studies on barklike species. Journal of the Lepidopterists' Society, 23(1), 1-9.

Scott, J. (1992). The Butterflies of North America: A Natural History and Field Guide. California, EUA: Stanford University Press.

Silva, A.R.M., Pontes, D.V., Guimaraes, M.P., Oliveira, M.V., Assis, L.T.F., \& Uehara-Prado, M. (2015). Fruit-feeding butterflies (Lepidoptera: Nymphalidae) of the área de Proteção Especial Manancial Mutuca, Nova Lima and Species list for the Region of Belo Horizonte, Minas Gerais, Brazil. Biota Neotropica, 15(3), 1-9. DOI: 10.1590/1676-06032015011814

Thiele, S.C., Milcharek, O., dos Santos, F.L., \& Kaminski, L.A. (2014). Butterflies (Lepidoptera: Hesperioidea and Papilionoidea) of Porto Mauá, Upper Paraná Atlantic Forest Ecoregion, Rio Grande do Sul State, Brazil. Biota Neotropica, 14(2), 1-10. DOI: 10.1590/1676-06032014000613

Thomas, C.D. (1991). Habitat use and geographic ranges of butterflies from the wet lowlands of Costa Rica. Biological Conservation, 55(3), 269-281. DOI: 10.1016/0006-3207(91)90032-5

Vasquez, J., Lamas, G., Couturier, G., \& Mejia, K. (2012). Aspectos biológicos de Panacea prola amazonica (Fruhstorfer) (Lepidoptera: Nymphalidae), en la Amazonia peruana. Folia Amazonica Amazónica, 21(1-2), 71-76.

Vasquez, R., \& Rojas, R.D.P. (2016). Clave para identificar grupos de familias de Gymnospermae $y$ Angiospermae del Perú. Missouiri, EUA: Missouiri Botanical Garden.

Yack, J.E., Otero, L.D., Dawson, J.W., Surlykke, A., \& Fullard, J.H. (2000). Sound production and hearing in the blue cracker butterfly Hamadryas feronia (Lepidoptera, Nymphalidae) from Venezuela. Journal of Experimental Biology, 203(24), 3689-3702.

Young, A.M. (1974). On the biology of Hamadryas februa (Lepidoptera: Nymphalidae) in Guanacaste, Costa Rica. Zeitschrift Für Angewandte Entomologie, 76, 380-393. DOI: 10.1111/j.1439-0418.1974.tb01897.x 